\title{
Indagaciones teórico-metodológicas sobre la construcción de ciudadanía cultural de comunidades de origen inmigrante en la Provincia de Río Negro (Argentina)*
}

\author{
A theoretical and methodological analysis of the construction of \\ Cultural Citizenship in communities with an immigrant background \\ in the province of Rio Negro (Argentina)
}

\author{
Patricia Dreidemie** \\ Eduardo J. Vior ${ }^{* * *}$
}

RESUMEN

A partir de una investigación que desde una aproximación intercultural a los derechos humanos estudió el ejercicio de ciudadanía de comunidades de origen inmigrante y sus efectos sobre la estructura del Estado y las políticas públicas en la Provincia de Río Negro (Argentina) en esta contribución se reflexiona sobre las herramientas teórico-metodológicas más aptas para este tipo de trabajo de campo. Parte de considerar que: 1- la inmigración modifica la noción de ciudadanía de las sociedades receptoras; 2- la ciudadanía siempre está culturalmente connotada, y 3- la coexistencia de colectivos etnoculturales heterogéneos en una misma geografía exige desarrollar el concepto de "ciudadanía cultural". El análisis se concentra en el rol de los "mediadores": personas o grupos sociales capaces de "traducir" en demandas políticas (y/ o en medidas de gobierno) las necesidades insatisfechas de las minorías en relación con sus derechos humanos. Estas figuras amplían el espacio público de comunicación al vincular, recrear y resignificar los códigos simbólicos de las minorías en interacción con los del Estado.

PALAVRAS-CHAVE: Derechos humanos e interculturalidad. Ciudadanías interculturales emergentes. Zonas de traducción. Inmigración.
The article introduces a research on citizenship practices of immigrant communities in Río Negro province (Argentina), and their consequences on public federal and state policy. The goal is to develop theoretical and methodological tools for fieldwork within an intercultural approach to Human Rights. It considers that: 1- immigration modifies citizenship notions of the host society; 2citizenship has always a cultural connotation; and, 3- the coexistence of diverse ethno-cultural social groups in the same location demands a reevaluation of the concept of "cultural citizenship". The analysis focuses on the role of the "mediators": persons or social groups capable of "translating" the need of the minorities' rights on politic demands (and/or government rules). These figures expand the communication field linking, recreating and resignifying the minority's symbolic codes in their interaction with the ones of the State

KEY-WORDS: Human Rights - Intercultural Studies. Intercultural Emergent Citizenship. Immigration.

\footnotetext{
*Proyecto de Investigación 25/09 financiado por la Universidad Nacional de Río Negro, Secretaría de Investigaciones, Desarrollo y Transferencia de Tecnología, por res. № 353/09 para el período 20092010.

** Doctora en Lingüística Antropológica (Universidad de Buenos Aires), Magíster en Análisis del Discurso (FFyL-UBA). Docente e investigadora de la Universidad Nacional de Río Negro (UNRN), Sede Andina (Bariloche, Argentina), patriciadreidemie@gmail.com.

***Doctor en Ciencia Política (Univ. de Giessen, Alemania, 1991), Mg. en Ciencia Política (Univ. de Heidelberg, Alemania, 1984), Prof. de Historia (Univ. de Buenos Aires, 1977), Profesor adjunto con dedicación completa en la Universidade Federal da Integração Latino-Americana (UNILA), Área de Política e Sociedade de América Latina, ejvior@gmail.com.
} 
The concept of people is not 'given', as an essential, class-determined, unitary, homogeneous part of society prior to a politics; 'the people' are there as a process of political articulation and political negotiation across a whole range of contradictory social sites. The people' always exist as a multiple form of identification, waiting to be created and constructed (HOMI BHABHA, 1990, p. 220).

Este artículo resume el marco teórico y metodológico del proyecto de investigación sobre Condiciones y consecuencias políticas e institucionales de la participación ciudadana de las comunidades de origen inmigrante en la Provincia de Río Negro desde una aproximación intercultural a los derechos humanos que E.J . Vior con la colaboración de P. Dreidemie y otras/ os investigadoras/ es llevaron adelante en la Universidad Nacional de Río Negro (Patagonia Norte, Argentina). ${ }^{1}$ Este proyecto se propuso indagar desde una perspectiva intercultural de los DDHH las condiciones de participación ciudadana de las comunidades de origen inmigrante y sus efectos sobre la estructura del Estado y las políticas públicas en la Provincia de Río Negro. Si bien la investigación debía durar un año, no fue completada por razones de financiamiento y por el pase del director a otra universidad, de modo que sólo duró nueve meses (abril/diciembre de 2009). Por estas razones no se presentan aquí conclusiones del trabajo de campo. ${ }^{2}$ A pesar de estas adversidades los autores consideran que el marco teórico-metodológico de la investigación construido en la primera etapa del mismo constituye un hito en las investigaciones sobre este tipo de procesos que puede ser aprovechado en muchos contextos similares. Por este motivo lo colocan a discusión de la comunidad científica internacional.

La problemática planteada intercepta conceptos complejos: "inmigración", "ciudadanía y formas de participación ciudadana”, "construcción nacional", "interculturalidad", "derechos humanos". Si bien cada uno de ellos se perfila someramente a lo largo del trabajo, aquí concentramos la atención en esclarecer el carácter de la(s) "zona(s) comunicacional(es) intercultural(es)” y sus dinámicas.

A través de las formas interaccionales listadas, a la vez materiales y simbólicas, el Estado regula - parafraseando a Taylor (1994) - las políticas del reconocimiento y subjetivación. Estos mecanismos de regulación son aplicados implícita o explícitamente según las constelaciones de fuerzas. La enunciación (o el anuncio) de reconocimientos sirve a veces como señuelo para perpetuar la subordinación. Otras veces hay comunidades que imponen reconocimientos tácitos. El mapa de los reconocimientos en el límite del Estado y las minorías etnoculturales no es sistemático, sino contingente y

\footnotetext{
${ }^{1}$ Proyecto UNRN 25/ 09.

2 Iniciado en Bariloche, el Alto Valle (Municipio de Cervantes) y el Valle Inferior del Río Negro.
} 
con límites fuertemente oscilantes. Con estas oscilaciones las burocracias estatales buscan 'asegurar' por medio de la marcación de los 'otros' y la asignación a ellos de lugares 'desplazados' la 'normalidad' de la (siempre en construcción e ilusoria) 'homogénea identidad nacional' y sus índices 'naturales' de estilo-de-vida (lengua, vestimenta, música, ocupación, comidas, color de piel, etc.). ${ }^{3}$

Durante las discusiones cuyas conclusiones aquí se presentan se analizó si la(s) “zona(s) comunicacional(es)” de interacción entre los inmigrantes y el Estado -vistas como conflictivos 'tableros de juego' (para retomar la clásica metáfora de Wittgenstein)- logran emerger en el campo como multidimensionales (bi- o multipolares) mixtas o sincréticas o, si el único resultado que el campo político (i.e., nacional, provincial y municipal) habilita sigue siendo que las diferencias culturales se sometan. En otras palabras la construcción de categorías estuvo concentrada en las condiciones para el reconocimiento de derechos de diversos grupos etnoculturales procedentes de países limítrofes (en especial de Bolivia, Paraguay y Chile) en aquellos espacios sociales 'fronterizos' surgidos de la interacción con la mayoría social de raigambre europea y las instituciones estatales argentinas que 'ordenan' (en el sentido foucaultiano) la escena nacional (e.g., oficinas de migraciones, policía, sistema educativo, sanitario, judicial, oficinas de fiscalización laboral o productiva, políticas demográficas).

Desde el inicio no se nos escapa que el campo investigado está delimitado desde hace más de cien años por el choque y la confrontación entre la colonización blanca y los grupos indígenas (preponderantemente mapuches) que resisten y se reconstituyen desde el aniquilamiento material y simbólico de su cultura. Esta confrontación, a duras penas silenciada por la cultura dominante, es determinante de todas las relaciones interétnicas y de la construcción de sociedad en toda la provincia. Este conflicto omnipresente es el que verdaderamente constituye la Patagonia como "zona de frontera". Sin poder abarcar el complejo de relaciones entre el Estado, la mayoría social, las comunidades originarias y las inmigrantes procedentes de los países limítrofes, en este proyecto se encararon las relaciones entre el Estado y la mayoría social de un lado y las comunidades latinoamericanas del otro en tanto condiciones de

3 Siguiendo a Hall (1986) se sostiene aquí que la marcación étnica de los grupos es flexible y dinámica en relación con luchas de poder, prestigio o privilegio situadas y no fijas o dadas ahistóricamente como el discurso dominante intenta imponer. En un Estado fuertemente fragmentado como el argentino, además, las luchas interburocráticas y las complejas alianzas entre sectores burocráticos, asociaciones formales o informales y organizaciones de la sociedad redundan coyunturalmente en avances tácticos de las comunidades de origen inmigrante que son difícilmente sistematizables. 
la construcción y ejercicio de ciudadanía, sin perder empero de vista que estas relaciones se inscriben en las condiciones particulares de la frontera. ${ }^{4}$

Aquí se sistematizan conceptualizaciones contemporáneas que focalizan "zonas de contacto, pasaje o conflicto" cultural, para avanzar críticamente sobre las diferentes aproximaciones (alternativas o complementarias) que abordan la "zona comunicacional fronteriza" referida. Para ello se revisan los conceptos de "zona de traducción" (FORNET-BETANCOURT, 2003), “sincretismo lingüístico” (HILL; HILL, 1999), “desplazamiento discursivo" (HAMEL, 1996), "liminaridad” (TURNER, 1980), "zona de contacto" (PRATT, 1997) y, finalmente, "traducción cultural" e "hibridación" (BHABHA, 1990, 1994).

El análisis epistemológico práctico que se presenta busca avanzar en la formulación de herramientas que habiliten el estudio de la formación de (algunas de) las nuevas subjetividades políticas surgidas del asentamiento perdurable y la articulación de las comunidades de origen inmigrante latinoamericano con el Estado y la mayoría social en zonas de frontera ${ }^{5}$. En particular interesa indagar la emergencia de "mediadores entre las culturas": personas y/o grupos capaces de "traducir" en demandas las percepciones y representaciones de las minorías sobre sus carencias de derechos humanos y otorgarles carácter político. Estas figuras vinculan (aún resignificando) los códigos simbólicos del Estado y la mayoría social por un lado y de los grupos subordinados procedentes de la inmigración latinoamericana por el otro, instalándose como piezas fundamentales en el escenario político estudiado. Entre estos grupos algunos entran en la interacción articulando códigos comunicativos y formas de participación colectiva históricas y tradicionales, como es el caso de los miembros de la comunidad boliviana que se reconocen como de origen indígena.

En resumen, enlazando aportes provenientes de la teoría cultural post- y decolonial (SAID, 1978; BHABHA, 1990, 1994; SPIVAK, 1990; MIGNOLO, 1993), la Filosofía Intercultural (BONILLA, 2010), la Antropología Lingüística (DURANTI, 2000; HYMES, 1972), la Lingüística de Contacto y Areal (THOMASON; KAUFMAN, 1988; CAMPBELL, 2006), la Antropología Sociocultural (ALONSO, 1994; GUPTA; FERGUSON, 1992; YUVAL-DAVIS, 1997; SEGATO, 2007; BRIONES, 1998) y los estudios politológicos y sociológicos sobre la participación política de comunidades de

\footnotetext{
${ }^{4}$ Para ello la investigación se apoyó en la combinación pragmática de métodos tomados de distintas disciplinas (ciencias políticas, análisis del discurso, lingüística antropológica, geografía cultural, etc.).

${ }^{5}$ Los autores no tienen experiencia de campo en la investigación de las relaciones entre otras comunidades de origen inmigrante subalternas y el Estado (i.e. comunidades china, africanas, etc.). Por eso se limitan a referir las categorías propuestas a las interacciones entre las comunidades de origen latinoamericano y el Estado, pero no descartan que - con las debidas adaptaciones- también puedan ser aplicadas al estudio de las reivindicaciones de derechos de otras comunidades.
} 
origen inmigrante (BOUCHARD; TAYLOR, 2008; D'AMATO, 2001; DUYVENE DE WIT; KOOPMANS, 2001; KOOPMANS, 1999, 2000; VIOR, 2006, 2007a, 2007 b), el objetivo de este recorrido es diseñar un modo de abordaje práctico e interdisciplinario que, sin perder de vista la conflictividad sociopolítica de las mediaciones (FOUCAULT, 1970), el carácter imaginado de los agrupamientos (ANDERSON, 1983) (no por ello menos operantes y específicos) ni la producción de otredades históricamente contextuadas (WILLIAMS, 1997, BOURDIEU, 1985; TODOROV, 1992), se perfile útil al trabajo de campo que enfrentamos.

\section{Proyección política de la investigación}

En general el estudio de la participación política de comunidades de origen inmigrante pone en cuestión los presupuestos sistémicos y normativos de la Ciencia Política en particular y de las Ciencias Sociales en general, porque investiga las condiciones bajo las cuales nuevos actores no previstos dentro del juego sistémico (y, en principio, no queridos) se incorporan al mismo, cómo modifican las reglas de juego y ponen en cuestión los supuestos mismos del Estado democrático colocándolo ante la opción de adaptarse a los cambios o cerrarse, perdiendo calidad democrática (VIOR, 2010a; 2010b). Planteado así, el estudio buscó poner de relieve las condiciones de formación de la ciudadanía democrática analizadas desde los bordes del sistema: en el contexto de un área de frontera y choque como es la Provincia de Río Negro.

En los países periféricos del sistema mundial como Argentina, en los que el Estado fue primero una construcción de elites coloniales o postcoloniales, la diferencia de códigos culturales, éticos y normativos entre quienes están adentro y los que están afuera de la "matriz nacional" (SEGATO, 2007) no es de grados o modos de participación en códigos culturales que se imponen, sino cualitativa (BHABHA, 1994; SPIVAK, 1990). En estos países, en los que predominantemente los estados nacionales se construyeron contra las mayorías originarias, primaron las relaciones de dominación, mientras que los consensos se construyeron o intentaron construir mediante la devaluación, supresión de identidad y silenciamiento de las culturas sometidas. En el marco de una heterogeneidad cultural constitutiva el acceso al ejercicio de derechos ciudadanos por parte de los sectores subordinados debió ser conquistado con mucho esfuerzo. El precio de la conquista fue muchas veces el tener que someterse a diversos mecanismos de homogeneización cultural. El resto de los sectores subordinados, en tanto, fue excluido de la formulación de demandas y la negociación de respuestas efectivas. Da la impresión que hoy en día la verbalización de las necesidades insatisfechas en materia de derechos humanos de los grupos 
minorizados, como de las comunidades que inmigran desde países limítrofes, pueblos originarios, refugiados, etc. y la negociación de las consecuentes demandas políticas no caben dentro de los códigos simbólicos de la cultura dominante (su "grilla" cultural), (BHABHA, 1990, p. 298). Más allá de las intenciones de mandatarios y funcionarios, el Estado sigue promoviendo un tablero de juego que coloca a las minorías de origen migratorio latinoamericano 'fuera' de la interacción. No los reconoce como interlocutores válidos, silenciando su presencia pública, folklorizándola o estigmatizando los mestizajes ${ }^{6}, 7$

El escenario político internacional actual en el que los grupos migratorios ${ }^{8}$ ponen en cuestión la naturalizada ecuación Estado-Nación-territorio, quebrando el vínculo lugar de residencia-etnicidad y constituyendo a diario etnicidades o asociaciones nuevas (e.g., denominados como 'grupos oprimidos', 'pobres', 'productores rurales'), plantea desafíos teóricos con consecuencias prácticas inmediatas: ¿cómo pensar estrategias de interlocución entre actores estructuralmente heterogéneos que permitan el reconocimiento mutuo como sujetos de derechos?; ¿hasta qué punto los grupos minorizados se comprenden por separado de las políticas públicas que los afectan?, ¿hasta qué punto es efectiva la representación de las diferencias y las jerarquías que (re)producen tanto los actores estatales de la mayoría social como los representantes de las minorías de origen inmigrante para alcanzar los respectivos objetivos?, y finalmente, ¿qué alcances tiene lo que Moreau llama (1984) "desdoblamiento de la subjetividad" de estos grupos subordinados a los que "se les exige a la vez identificarse con el grupo dominante y disociarse de él” (apud PRATT, 1987)?

En este punto el análisis de perspectiva comunicacional puede contribuir a: 1- el avance del conocimiento crítico de los mecanismos funcionales a la dominación colonial, 2- deconstruir formas de regulación social consolidadas mediante el uso diferencial de códigos simbólicos, 3- descentrar los esencialismos y focalizar

${ }^{6}$ El Estado se ocupa de que lo propio de los grupos étnicos subordinados sea fijado en una distancia temporal y espacial importante. Cualquier transformación vital de los prototipos impuestos (su fosilización) será acusada de 'pérdida de la pureza original'. Entre otros derechos negados, los grupos minorizados parecen no tener acceso al "derecho al cambio": la movilidad sociocultural que indexa su carácter vital. Por supuesto que los grupos afectados producen diariamente numerosas y creativas estrategias de resistencia (KULICK, 1992; REGALSKY, 2005; HALPERN, 2005; DREIDEMIE, 2006). Bajo la necesidad de articularse contextualmente con la mayoría social desafían de diversos modos las matrices témporo-espaciales nacionales (a través de mitologías contrahegemónicas, resignificación de recursos, recontextualización del discurso dominante, etc.).

7 Es útil en este contexto utilizar el concepto de maquinarias de territorialización ("territorializing machines") desarrollado por Grossberg (1992) quien, tomando como base la teoría Foucaultiana (1970, 1973, 1985), las define como procesos de construcción de regímenes de jurisdicción o poder que sobre una dinámica conflictiva y a partir de habilitar "modelos de identidad y diferencia social" pre-escriben lo que los sujetos pueden hacer (sus "mapas de posibilidades"), sus procedimientos y orientaciones.

8 'Flujos' u 'olas migratorias', según las metáforas acuáticas tan recurridas para designarlos. 
particulares esferas y prácticas de interacción contextuadas y 4- dar cuenta de la aparición de nuevas subjetividades en la escena política.

En la dimensión política esta línea de investigación se propone investigar las condiciones de construcción de las "ciudadanías interculturales emergentes" (BONILLA, 2005, 2008; VIOR; BONILLA, 2008). Esta categoría parte de considerar: 1- que por pequeña que sea la inmigración, confronta la sociedad receptora con la necesidad de adaptar su noción de "ciudadanía”, por lo que se convierte en un tema transversal a toda la política del Estado; 2- que el ejercicio de la ciudadanía nunca es culturalmente neutro y 3- que la coexistencia de individuos y de grupos de procedencia migratoria de gran heterogeneidad, interactuando en relaciones dinámicas con las sociedades “de acogida” y entre sí, exige que se incluya la categoría de "ciudadanía cultural" (que involucra idiomas, costumbres, moralidades y sistemas simbólicos) y el carácter inestable y abierto de las relaciones. Al rotular la problemática como "ciudadanías interculturales emergentes", Vior y Bonilla han procurado hacer visibles los modos diferenciados de subjetivación política, generación de poder y participación ciudadana que surgen de la interacción (libre o más o menos compelida) entre los grupos y las personas en el marco de la pluralidad de las sociedades sudamericanas actuales. En Argentina, más acá de lo exigido por la norma migratoria, los modelos actualmente vigentes de tratamiento estatal de las comunidades de origen inmigrante actualizan intentos de homogeneización cultural que atentan contra la consideración como "sujetos de derechos" de las personas de origen migrante o pertenecientes a minorías étnicas o lingüísticas, creando a la vez zonas de indocumentación e ilegalidad que impiden al Estado cumplir con las funciones ordenadoras que públicamente se le reclaman y un Estado democrático debe cumplir.

A partir de estas evaluaciones diagnósticas esta línea de investigación se orienta a verificar dos hipótesis interrelacionadas:

1. Que sólo la presencia de "agentes mediadores entre las culturas" con capacidad para traducir códigos simbólicos entre las comunidades de origen inmigrante, la mayoría social y el Estado puede permitir "traducir" en demandas las percepciones y representaciones de estas minorías excluidas sobre sus carencias de derechos humanos y dar a las demandas un carácter político.

2. Que si los representantes y funcionarios estatales persisten en ignorar las carencias y/o las demandas de las comunidades de origen inmigrante, la progresiva pérdida de representatividad estatal afectará su eficacia y capacidad de gobernabilidad. 


\section{Interculturalidad y territorio(s): de la "zona de traducción" al "espacio liminal”}

La migración, como una de las materializaciones del intercambio entre culturas, diseña una "zona de contacto" que de alguna manera mide la capacidad de cada sociedad para tratar con los otros' culturales. Para analizar la "zona comunicacional fronteriza" que se investiga aquí, es clave y articulador el concepto de "zona de traducción" desarrollado, desde la Filosofía Intercultural por Fornet-Betancourt (2003) quien asume la batalla de "des-occidentalizar", "regionalizar", "mundanizar" y "contextualizar" el pensamiento filosófico contemporáneo cuyas premisas - entiendeestán siendo desafiadas por la ubicua y cada vez más visible interculturalidad. Este concepto y otros que se revisarán críticamente a continuación configuran el conjunto de herramientas teórico-metodológicas que se han reunido para investigar en terreno las formas en que se expresa lo que Vior y Bonilla (2008) han denominado "ciudadanías interculturales emergentes".

Fornet-Betancourt (2003) denuncia en numerosos trabajos cómo en el marco de los Estados liberales la condición de "ciudadanía” se levanta como una frontera que controla, regula e inhibe la vida de los inmigrantes. ${ }^{9}$ Para esta investigación se retoma el "modelo de la traducción" por él propuesto (en congruencia con autores que le sirven de fuente: Ricoeur, Benjamin, etc.). Según Fornet-Betancourt es en las "zonas de traducción" (de dimensiones pragmáticas y hermenéuticas) donde los intérpretes construyen valores emergentes a través de la interrelación dialogal de sus respectivos mundos culturales. En este proceso los referentes de cada uno y sus universos discursivos, al comprenderse de acuerdo a la metáfora de la traducción, "sufren" una natural pérdida de exactitud, pero a la vez "ganan" en el nivel de la interpretación, la crítica y la coproducción del conocimiento. La perspectiva filosófica intercultural de Fornet-Betancourt proyecta la posibilidad de una "comunidad abierta" a la construcción dialógica del sentido, con participantes de los más variados niveles y

\footnotetext{
9 Fornet-Betancourt (2003) propone que: “desde la barrera de la ciudadanía se legaliza e institucionaliza la exclusión de los inmigrantes porque con la ciudadanía se invierte o, mejor, se pervierte el orden ético condensado en el principio de igual dignidad de los seres humanos al hacer del reconocimiento como ciudadano por parte del Estado la condición indispensable para tener acceso y poder disfrutar de los derechos del ser humano"; "esta ciudadanía excluyente es un poderoso instrumento de selectividad y control", que, por un lado, "promueve, desde una ciudadanía entendida como culturalmente homogénea, que a los inmigrantes se los vea y trate como 'invasores' que hay que neutralizar"; y, por el otro lado, que "se contradiga la constitución pre-política de la dignidad humana”.
} 
procedencias culturales. ${ }^{10}$ Destaca la ganancia que la complementación implica y la puesta en discusión de lo "normal" en base a aportes de autores y actores provenientes de otros consensos y regiones. En este sentido, según el autor, lo que el discurso del poder (i.e., de la globalización) dificulta u obstruye es ese espacio dialógico de producción de verdades y saberes que el pensamiento intercultural (con el concepto levinasiano de "alteridad" como principio de progresiva y decisiva incidencia en una renovada comprensión del espacio público), pretende liberar. Se trata de una perspectiva receptiva y crítica de ciudadanía que asume posiciones combativas y utópicas.

El siguiente concepto que corresponde revisar es el de "sincretismo lingüístico" propuesto por J ane Hill y Kenneth Hill (1999) en Hablando mexicano; La dinámica de una lengua sincrética en el centro de México ${ }^{11}$. Los autores conciben el sincretismo lingüístico como una forma de referir fenómenos de "mezcla" en los modos de expresión sin arrastrar las connotaciones viciadas que la teoría generativa y purista le había impreso. El concepto designa "el fenómeno lingüístico que se nutre de una variedad de materiales simbólicos de distintas lenguas" (HILL; HILL, 1999, p. 73) y se interpreta como parte de un proyecto de manipulación de los materiales simbólicos (en los que se incluye/ $\mathrm{n}$ las lenguas) para crear nuevas formas transitorias de participación en la interacción social relativas a contextos dinámicos. La conceptualización reconoce la negociación activa de los hablantes y no concibe "los puentes" comunicacionales construidos por los migrantes como un síntoma de degeneración sino como un signo de la vitalidad y adaptabilidad fundamental de sus tradiciones. Para Hill y Hill el proyecto sincrético pone en evidencia la retención, continuidad y productividad de dispositivos vernáculos o patrones de uso que son re-creados por los migrantes estratégicamente para asegurar la supervivencia del grupo. A través de re-significar oposiciones en diferentes niveles semióticos, estos mecanismos conforman "estrategias defensivas y de solidaridad" frente a la sociedad mayor que posibilitan controlar el acceso a recursos comunitarios en el marco de una "economía de mercado" que condiciona los valores en juego (HILL; HILL, 1999; HILL, 1993).

10 Una de las tareas programáticas que Fornet-Betancourt reconoce en el marco de la Filosofía intercultural actual es la labor de interpretación y comprensión de las "superposiciones" culturales que, según él, debería efectuarse sobre una actitud "hermenéutica intercultural". Ella derivaría, entre otros elementos, en el desarrollo de una teoría política hacia el extranjero que supere las prácticas estatales actuales (que sólo promueven su asimilación e integración), los "autorice" como "iguales pero diferentes" y redefina las fronteras del orden jurídico e institucional establecido (FORNET-BETANCOURT, 2003).

${ }^{11}$ El análisis conceptual y crítico que sigue retoma parte de lo desarrollado por Dreidemie (2006). Desde 2001 la autora documenta y analiza las prácticas comunicativas de los migrantes quechuabolivianos en contextos urbanos y semiurbanos de Argentina. 
$\mathrm{Si}$ bien esta conceptualización recupera la mirada sobre el proceso de reformulación cultural entre dos códigos, con la que "ambas partes de la ecuación resultan modificadas", es decir, donde emerge "una nueva realidad compuesta y compleja; una realidad que no es una aglomeración mecánica de caracteres, ni siquiera un mosaico, sino un fenómeno nuevo, original e independiente" (MALINOWSKY, 1985), su apropiación requiere de ciertos cuidados. Por un lado el concepto de "sincrético" traslada el presupuesto de preexistencia ontológica y polarizada (esencialista) de códigos paralelos, autónomos, contrastantes y simultáneos que entran en relación. Por otro lado se posiciona frente al peligro de neutralizar la dimensión del poder - económico, político y social- y del conflicto por la hegemonía (WILLIAMS, 1997; BOURDIEU, 1993) dentro de un marco implícito de armonía. De todas formas posee varias ventajas por su visión valorizante de las formas expresivas emergentes y porque re-integra a través de la metodología etnográfica las prácticas etnoculturales con su naturaleza histórica y social. En esta investigación se propone avanzar un paso más, aplicando esta conceptualización a los intercambios discursivos entre las comunidades de origen inmigrante, mandatarios, funcionarios y representantes de instituciones sociales mayoritarias, para indagar en qué medida en las "áreas de contacto" se producen resignificaciones de ideologemas relativos al poder, la dominación y el reconocimiento mediante fenómenos de sincretismo.

Los trabajos de Hamel $(1995,1996,1997)$ también son reveladores de una nueva perspectiva de análisis sobre el fenómeno comunicacional de contacto. Él propone un marco teórico socio-lingüístico en el que se incluyen fundamentalmente el aspecto pragmático, metalingüístico y las relaciones ideológicas de las prácticas interaccionales interculturales. El modelo interrelaciona tres niveles en los que surgen (dis)continuidades, combinaciones y "cruces de fronteras" en el proceso de comunicación: el nivel de los modelos culturales, el de las estructuras discursivas (incluyendo los procedimientos de contextualización y de inferencia) y el de las estructuras lingüísticas (propiamente, el nivel del código lingüístico). Los tres niveles forman parte de un mismo sistema semiótico de "desplazamiento discursivo" que se concibe en proceso de cambio y que adquiere diversas formas. En oposición a lo que habitualmente realizan los estudios sobre el desplazamiento cultural, Hamel enfatiza el carácter de las prácticas comunicativas no sólo como recursos de comunicación sino principalmente como instrumentos de poder social relacionados con la identidad de las comunidades que interactúan. Según Hamel, frente a la dinámica dialéctica establecida entre dos tendencias fuertes (la expansión de una cultura dominante junto a sus normas de comunicación hegemónicas y su mundo simbólico y el desplazamiento 
progresivo de una minorizada de sus usos y prácticas tradicionales) se producen en la comunidad "desarticulaciones discursivas y culturales" que por un lado transforman las bases interpretativas del grupo (interfieren en sus modelos culturales y fomentan las contradicciones) y por el otro impulsan nuevas tendencias re-organizativas de las prácticas interaccionales, modificando paulatinamente la relación entre los recursos expresivos en uso y la experiencia acumulada a través del tiempo por los participantes (HAMEL, 1996). Según el autor, la "ruptura inicial" y la reorientación discursiva de las personas manifestaría estrategias de re-territorialización (GROSSBERG, 1992; FOUCAULT, 1985) y apropiación particulares del nuevo espacio simbólico.

Sin embargo, el marco de análisis propuesto por Hamel de alguna forma también arrastra ciertos presupuestos que es necesario considerar críticamente. La definición de "desplazamiento" cultural conlleva la idea de un proceso transitivo desde una cultura hacia otra, una especie de pasaje que implica pérdida y desarraigo (parcial deculturación) así como la conformación creativa de nuevos fenómenos (neoculturación) en la incorporación de otro código dentro del contexto inmigratorio. Es decir, al igual que el concepto de sincretismo presupone la existencia primordial de dos culturas como entidades autónomas y homogéneas que se ponen en contacto y naturaliza un pasaje unidireccional que mediante la metáfora espacial configura zonas claramente diferenciables entre las cuales el espacio fronterizo es sólo transitorio. Por el contrario, desde una perspectiva sincrónica las culturas no pueden ser pensadas como entidades autónomas, por un lado, en tanto se (in)definen relacionalmente (dinámica y conflictivamente) y, por el otro, porque considerarlas así reproduce una perspectiva ajena a la praxis individual y colectiva. Desde una perspectiva diacrónica no puede dejar de considerarse el valor de los fenómenos observados que neutralizan siglos de "contacto", lucha y mutua (in)determinación. La aproximación de Hamel presupone también un cierto equilibrio funcional entre las tres variables estudiadas que de forma natural tendería a reestablecerse una vez asimilada la ruptura inicial. En términos de García Canclini (1992), una especie de "ecualización”. Este último autor reproduce con la metáfora musical la idea de una hibridación tranquilizadora que reduce los puntos de resistencia de otras culturas. El marco "pluralista" de la globalización, bajo la forma del "multiculturalismo", de alguna manera, actualiza políticamente esta metáfora. ${ }^{12}$

En esta investigación se toma distancia de estas perspectivas, al no retomar la idea de una presupuesta congruencia resultante que vuelve a normalizar matrices

12 Bonilla (2008) - así como otros investigadores- señala la deficiencia de las teorías denominadas "multiculturalistas" y de las políticas multiculturales. 
hegemónicas actualizando significados implícitos de armonía, polifonía o intercambio cultural. La concepción que aquí se aplica también se diferencia de la construcción de "frontera" que los autores referidos diseñan: no se la entiende como línea' sino como 'espacio', un "espacio de frontería” (DREIDEMIE, 2006a) o territorio (físico y simbólico) particular en el que el carácter inestable no conforma un estado circunstancial de tránsito sino que es constitutivo de su condición de ser.

En un aspecto se retoma la idea de desarticulación cultural por contacto: por un lado, las estrategias discursivas de los representantes del Estado en sus intercambios con las comunidades de origen inmigrante están predominantemente orientadas a que éstas reproduzcan los códigos dominantes o callen. Sin embargo, la realidad muestra un abanico mucho más amplio de intercambios en los que, si bien subordinados, los grupos de origen inmigrante distan de ser pasivos e interpelan activamente al Estado y sus representantes. Por otro lado, es posible prever la posibilidad de que también los discursos y usos dominantes resulten desarticulados por las demandas silenciosas o verbalizadas de estas comunidades y comiencen a articularse con ellas creando nuevas hibridaciones que introducen conflictos dentro del mismo Estado. Estos efectos discursivos sobre el Estado también deben ser materia de investigación.

En este sentido se rescata particularmente el concepto de "liminaridad" desarrollado por Turner (1980), un antropólogo dedicado a analizar el rol de la simbología, el ritual y la performance en la reproducción cultural y en el mantenimiento de la solidaridad social. Desde una conceptualización dialécticoestructural define la liminaridad como una zona que reúne intersticios y márgenes de la estructura, posicionamientos sociales que no participan del orden imperante y que se relacionan con los niveles inferiores de las jerarquías de poder. En lo liminar se constituye un espacio social móvil, de "pasaje" o periférico, desde donde se deconstruyen clasificaciones y se generan nuevos modelos/sistemas culturales capaces de re-configurar las relaciones sociales. Su aporte consiste en pensar la existencia de estos espacios sociales inestables en los que se producen movimientos que, a partir de desmontar estructuras hegemónicas en base a una dinámica de referencia y condensación, presuponen (y reproducen) tanto como (re)crean ciertos aspectos de la estructura. Desde posicionamientos no-centrales y "pasajeros" estos lugares hacen posible la reproducción e incluso la generación de valores, normas, prácticas, creencias e identidades que ponen en relación dialéctica patrones culturales del pasado con posicionamientos presentes. De esta manera la liminaridad conforma un "hábitat migrante" que, si bien periférico, puede desmontar estructuras de poder social. 
Esta noción junto a las siguientes habilitará a pensar las prácticas comunicativas "mezcladas" y emergentes ("contextualizantes") de poblaciones "marginales" por "migrantes" como formas que codifican procesualmente y dinámicamente una historia (política) de "textos interaccionales" (SILVERSTEIN, 1993) constituida por una profusión de relaciones sociales, identidades impuestas resistidas y subvertidas y representaciones del mundo que se repiten, re-producen, polemizan y cambian según intereses prácticos (in)mediatos. Los intercambios entre los representantes del Estado y de la mayoría social de un lado y de las comunidades de origen inmigrante del otro dejan por este medio de ser pensadas como alocuciones "por encima del cerco", para empezar a ser estudiados como constelaciones estratégicas y tácticas en las que se revaloriza la agencia de los subordinados, sin por ello perder de vista las estructuras de dominación existentes.

Concordante con lo anterior (y aún más flexible, en tanto no presupone una estructura social y simbólica global), se rescata el concepto de zona de contacto formulado por Mary Louise Pratt (1997). Proveniente de los estudios culturales, esta noción favorece el trascender los límites de las "comunidades culturales", en tanto concibe el espacio social en relación dialéctica con las prácticas y no a priori de ellas. Definido como "un espacio social en el que culturas diversas se encuentran y establecen relaciones duraderas de dominación y subordinación fuertemente asimétricas que implican coerción, desigualdad y conflicto", este concepto contribuye al desarrollo de una perspectiva sobre los procesos de transformación y cambio etno-cultural en las llamadas "minorías" desde una concepción dinámica de los recursos comunicacionales como prácticas sociales, históricas, emergentes, creativas y fundamentalmente relacionales.

Finalmente se retoman dos conceptos de Homi Bhabha (1990) (observaciones del autor motivadas por trabajos de Benjamin): primero, el concepto de "traducción cultural" a través del cual destaca que todas las formas de cultura están de cierto modo relacionadas entre sí, porque la cultura conforma una actividad simbólica y significante. En este sentido Bhabha propone que las culturas no son comparables por sus contenidos sino por sus "haceres": las prácticas interpretativas, la formación de símbolos y la constitución de sujetos. Al reunir y generar siempre formas de representación, ninguna es esencial en sí misma, sino que todas actualizan referentes proyectados, sosteniéndose sobre la actividad de desplazamiento inherente al signo lingüístico: una estructura descentrada, "liminal”, cuya dinámica está abierta a articular otras prácticas culturales y prioridades, dado que se basa en conjuntos interpretantes que se encuentran en procesos de transformación e hibridación continua. Y aquí el 
segundo concepto que se ha seleccionado de Bhabha, el de "hibridación" o "tercer espacio": aquél donde historias disímiles se articulan produciendo un escenario en el que nuevas iniciativas políticas, estructuras de autoridad y formas de identificación se construyen cotidianamente. El proceso de hibridación, según Bhabha (1990, p. 211), conforma un "área de negociación de significados y representaciones" que actualiza la "metáfora migrante": un conjunto de signos ambivalentes, dobles, disimulados y flexibles, aptos para asumir significados variables, cuando una nueva alianza o escenario reclama repensar, expandir o traducir principios, narrativas o tradiciones intelectuales. Esta noción conlleva pensar a las personas "siendo(se) construidas" a través de la marcación de las diferencias culturales y los procesos de hibridación constantes (por lo que cualquier descripción monolítica de poder autoritario queda invalidada). La aproximación se basa en un énfasis en la naturaleza ambivalente de las relaciones sociales, la multi-dimensionalidad de las subjetividades políticas y las conflictivas formas de identificación 'disponibles' en un contexto dado.

De diferentes modos los conceptos expuestos fundamentan la aproximación intercultural elegida y la detención en los marcos de interpretación en tanto recursos simbólicos imprescindibles para entender la lucha situada (espacial, territorial y jurisdiccionalmente) de las comunidades de origen inmigrante, para construir para sí identidad(es) útil(es) y organizar su(s) mundo(s) de tal manera de poder sobrevivir y prosperar en el(los).

\section{Enclaves de frontería: mediadores, ciudadanías emergentes, áreas comunicacionales liminares}

A partir de lo desarrollado quedan formuladas las siguientes observaciones $u$ orientaciones de trabajo:

a) Desde el punto de vista lógico existe una interrelación entre la capacidad de un grupo social de percibir sus carencias de derechos humanos, de decidir si las expresa o las calla por conveniencia o miedo y su habilidad para verbalizarlas, presentándolas como demandas. En cambio sus estrategias para darles un carácter político dependen no sólo de la existencia de "mediadores interculturales" capaces de traducir los códigos simbólicos entre el Estado y la mayoría social por un lado y los grupos subordinados por el otro, sino también de coyunturas específicas y de la destreza de los mediadores y conductores de los reclamos para presentarlos convincentemente a las instituciones receptoras como de un interés superior al sectorial (VIOR et al., 2009). Puede afirmarse que todo tipo de demandas de los grupos excluidos produce efectos sobre la estructura y las prácticas del Estado y la burocracia estatal, aun cuando los dirigentes 
políticos y funcionarios intenten minimizarlos y/ o negarlos. De la capacidad de estos últimos de registrar la aparición de nuevos actores en la escena política y consecuentemente de adaptar la estructura y las prácticas del Estado y la administración pública a los requerimientos que plantea el diálogo con ellos, depende la gobernabilidad y la eficacia del Estado.

b) La coexistencia en vastas regiones de las naciones actuales de individuos y de grupos migrantes de gran heterogeneidad, mayoritariamente pobres, interactuando en múltiples relaciones dinámicas con las sociedades denominadas de acogida y entre sí, impone por su propio peso una revisión más completa de la noción de ciudadanía en la que se incluya fuertemente la categoría de "ciudadanía cultural", pero a la vez el carácter inestable, dinámico y abierto de las relaciones referidas. Para abonar en defensa de este intento es posible pensar, como E. Vior lo ha propuesto en otro lugar (VIOR; BONILLA, 2008), en "Ciudadanías interculturales emergentes" "como facilitadoras de inclusiones no sesgadas por formas de dominación asimilacionistas o integracionistas, siempre y cuando la noción de 'interculturalidad' no se emplee aquí en el sentido corriente". Se adscribe así a una noción "fuerte" de interculturalidad que se basa en la idea de contextualidad no relativista de la razón y posibilita un "polílogo" entre las culturas, efectivizado en "zonas de traducción" mutuamente posibles (FORNET- BETANCOURT, 2003, p. 19). “Quizá sea ésta la única posibilidad, ya no de 'elegir' al ciudadano de un territorio, sino de que éste elija dónde y cómo convivir con sus semejantes, aunque haya arribado a él en condiciones de sometimiento, penuria y desigualdad" (VIOR; BONILLA, 2008).

c) Con el desafío de estudiar la zona "de frontera" o "de traducción" adoptando una perspectiva interdisciplinaria, se propone como clave para el análisis de este tipo de fenómenos el estudio de la dinámica de las prácticas comunicativas (BAUMAN; BRIGGS, 1996, HANKS ,1996), ya que ellas conforman un lugar privilegiado en el que se dirimen cuestiones de poder e identidad, se actualizan tensiones sociales y se (re)producen estrategias (meta)pragmáticas de resistencia cultural (DREIDEMIE, 2006b, 2008). En este sentido el foco se posiciona sobre la emergencia de formas públicas (en transformación) en las que experiencias colectivas construyen espacios discursivos comunes (lugares, posicionamientos, órdenes, desplazamientos y trayectorias posibles) de negociación, apropiación, dominación o subordinación cultural. Para ello se requiere un intenso trabajo de reconocimiento de sistemas de valores diversos, formas expresivas y marcos interpretativos generados a partir de historias socioculturales específicas por un lado y del análisis de los procesos de 
construcción y reconstrucción de significación en función de necesidades prácticas de las personas y escenarios locales por el otro.

d) Finalmente, como hipótesis para trabajos futuros se deja planteada la posibilidad de que las zonas comunicacionales fronterizas puedan ser analizadas como “áreas culturales" de rasgos específicos (DREIDEMIE, 2010a). Entre los diferentes tipos posibles éstas conformarían especies de "áreas liminares" que se definen como "espacios donde prácticas comunicativas ancladas en presupuestos culturales disímiles se encuentran, polemizan e interaccionan, promoviendo la (re)creación de formas expresivas que resultan constitutivamente complejas y afectan performativamente la trama social" (DREIDEMIE, 2010a). La urdimbre social, en principio, es compelida indirectamente a flexibilizar su grilla categorial - su "grid", según Bhabha (1990). En este sentido se considera que la diferenciación social (de carácter relacional y fragmentada) establece entre migrantes adscriptos a comunidades etnoculturales, la sociedad de acogida y las prácticas del Estado un área comunicacional liminar, con características propias y probable(s) juegos complejos de centro(s) y periferia(s).

Para la continuación de esta línea de investigación en el mismo o en otros contextos el objetivo sería estudiar los procesos que puedan conducir a la promoción de "interlocutores válidos entre el Estado y las comunidades de origen inmigrante": es decir personas o grupos que, adscriptas a alguna de las comunidades migrantes o a la administración del Estado -sin ser asimiladas ni ceder los presupuestos culturales vernáculos y/o hegemónicos ni sus marcos interpretativos-, sean competentes en escenarios comunicativos múltiples (BATESON, 1972; GOFFMAN, 1981; GUMPERZ, 1982), especialmente en aquellos "matriciados" bajo los modos interaccionales del Estado y/o los modos 'normalizados' (naturalizados y regulados) por las prácticas estatales, o sepan alcanzar las competencias por su ubicación coyuntural en el entramado de relaciones entre el Estado y estos grupos subordinados. Estos modos poseen características diacríticas que se reconocen rápidamente: entre otras la alta burocracia (la necesaria intermediación y las gestorías, la utilización de formularios, los procedimientos escalonados e indirectos), el valor del 'documento', la palabra escrita y el lugar del número, los rotulamientos arbitrarios e inmanentes a sistemas administrativos propios (sus funcionales binarismos: civil-estatal, público-privado), los des-tiempos, la estratégica des-información, la des-personalización, masificación o mitigación de particularidades. Son de destacar entre ellos las operativas (pre)clasificaciones sociales (por clase social, ocupación, género, edad, etnicidad, lugar/mapa de residencia, etc.), la fiscalización de la Historia (la construcción de la 'memoria común') y la habilitación y distribución de las tierras rurales y urbanas (con 
sus fronteras 'densas') que conforman la materialización de rutinas, rituales, vigilancias y protecciones selectivas de los organismos estatales sobre el cuerpo social. Estos controles son especialmente evidentes y funcionales 'frente' a los inmigrantes procedentes de países limítrofes quienes devienen los excluidos sistemáticos de estas lógicas de fragmentación y selección.

\section{Referência}

ALONSO, A. The Politics of space, time and substance: state formation, nationalism, and ethnicity. Annual Review of Anthropology, v. 23, p. 379-405, 1994.

ANDERSON, B. Imagined communities: reflections on the origin and spread of nationalism. Londres: Verso, 1983.

BATESON, G. Steps to an ecology of mind. Northvale (NV): Vason Aronson, 1972.

BAUMAN, R.; BRIGGS, C. L. Género, intertextualidad y poder social. Revista de Investigaciones Folklóricas, v. 11, p. 78-108, 1996.

BHABHA, H. The location of culture. New York: Routledge. 1994.

. The third space. Interview with. In:

(Ed.). Identity: community, culture, difference. London: Lawrence and Wishart, 1990. p. 207-221.

BONILLA, A. El diálogo filosófico intercultural y el fenómeno migratorio: su tratamiento como 'traducción' en la Escuela de Aachen. In: CONGRESO LATINOAMERICANO DE ANTROPOLOGÍA., 1., 2005, Rosario Actas... Rosario, 2005. CD-ROM, p. 29-47.

Imágenes de nación y ciudadanías interculturales emergentes. In: GUERCI $\overline{\mathrm{D}} \overline{\mathrm{E}} \overline{\mathrm{SI}} \overline{\mathrm{UFI}}$, Beatriz (Comp.). Filosofía, cultura y sociedad en el NOA. San Salvador de J ujuy: EDIUNJ u, 2008, p. 27-34.

. Vulnerabilidade vs. autonomia conflitos das migrações contemporâneas. Passagens: Revista Internacional de História Política e Cultura J urídica, v. 2, n.4, maio/ ago. 2010. p. 4-38.

BOUCHARD, G.; TAYLOR, C. Fonder l'avenir: le temps de la conciliation. Rapport. Bibliothèque et archive national de Québec, Gouvernment de Québec, 2008. Disponible en: <http:// www.accommodements.qc.ca/ documentation/ rapports/ rapport-finalintegral-fr.pdf>. Acceso en: 15junio 2009.

BOURDIEU, P. The social space and the genesis of groups. Social Science Information, v. 24, n. 2, p. 195-220, 1985.

. Cosas dichas. Madrid: Gedisa, 1993.

BRIONES, C. (Meta)cultura del estado-nación y estado de la (meta)cultura. In: SEMINARIO INTERNACIONAL. UMA AGENDA PARA A ANTROPOLOGÍA A PARTIR DA AMÉRICA LATINA, 1998, Brasília. Comunicación.. Brasília, 1998. Série antropología. 
CAMPBELL, L. Areal linguistics: a closer scrutiny. In: MATRAS, Y.; MCMAHON, A.; VINCENT, N. Linguistic areas: convergence in historical and typological perspective. Hampshire: Palgrove Macmillan, 2006. p. 1-31.

D`AMATO, G. Vom Ausländer zum Bürger: der streit um die politische integration von Einwanderern in Deutschland, Frankreich und der Schweiz. Band 5. Münster: LitVerlag, 2001.

DREIDEMIE, P. Espacio de frontería: campos sociales, trayectorias y prácticas posibles entre migrantes quechuas bolivianos residentes en Buenos Aires. In: CONGRESO LATINOAMERICANO DE EDUCACIÓN INTERCULTURAL BILINGÜE, 7, 2006, Cochabamba. Actas... Cochabamba: Ministerio de Educación y Culturas, 2006a.

Estrategias discursivas de persistencia cultural: la (dis)continuidad del quechua en el habla mezclada de migrantes bolivianos en Buenos Aires. 2006b.Tesis (Maestría en Análisis del Discurso). Universidad de Buenos Aires, Buenos Aires.

. Formas de poder y regulación social a través del habla: el caso de los migrantes quechua-bolivianos en Buenos Aires (Argentina). In: CONGRESO ARGENTINO DE ANTROPOLOGÍA SOCIAL FRONTERAS DE LA ANTROPOLOGÍA, 9, 2008, Posadas. Actas...Posadas, 2008a. CD-ROM.

D _ _._. Performatividad discursiva y espacio(s) social(es) liminar(es): una propuesta de aproximación a las prácticas comunicativas de migrantes indígenas quechua-bolivianos en Buenos Aires (Argentina). RUNA, Revista del Instituto de Ciencias Antropológicas, Buenos Aires: Facultad de Filosofía y Letras, Universidad de Buenos Aires, n. 28, 2008b.

. Estudio etnolingüístico del quechua hablado por migrantes bolivianos en Buenos Aires (Argentina). Tesis(Doctorado en Lingüística - Especialización Lingüística Antropológica) - Universidad de Buenos Aires - FFyL, Buenos Aires, 2010a.

. Lingüística de campo y traducción en la investigación sobre lenguas indígenas en Argentina: el caso de las comunidades migrantes de habla quechua y toba. In: COLOQUIO INTERNACIONAL "ESCRITURAS DE LA TRADUCCIÓN HISPÁNICA, 2., 2010, Bariloche. Actas...Bariloche: Universidad Nacional de Río Negro, 2010b.

DURANTI, A. Antropología lingüística. Madrid: Cambridge U.P, 2000.

DUYVENE DE WIT, T.; KOOPMANS, R. Die politisch-kulturelle Integration ethnischer Minderheiten in den Niederlanden und Deutschland. Forschungsjournal NSB, año 14, n. 1, 2001, p. 26-39.

FORNET-BETANCOURT, R. Supuestos, límites y alcances de la filosofía intercultural. BROCAR, v. 27, p. 261-274, 2003a.

. Interculturalidad y filosofía en América Latina. Aachen:

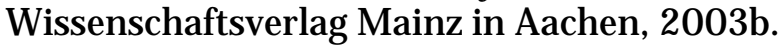

FOUCAULT, M. El orden del discurso. Barcelona: Tusquets, 1970.

La verdad y las formas jurídicas. Barcelona: Gedisa, 1973.

. ¿Qué es un autor? México: Universidad Autónoma de Tlaxcala, 1985. 
GARCÍA CANCLINI, N. Culturas híbridas: estrategias para entrar y salir de la modernidad. Buenos Aires: Sudamericana, 1992.

GOFFMAN, E. Forms of talk. Philadelphia: University of Pennsylvania Press, 1981.

GROSSBERG, L. We gotta get out of this place; popular conservatism and postmodern culture. New York, London: Routledge 1992.

GUMPERZ, J . Discourse strategies. Cambridge: Cambridge University Press, 1982.

GUPTA, A.; FERGUSON, J . Beyond culture: space, identity and the politics of difference. Cultural Anthropology, v. 7, n. 1, p. 6-25, 1992.

HALL, S. Gramsci's relevance for the study of race and ethnicity. J ournal of Communication Inquiry, v. 10, n. 2, p. 5-27, 1986.

HALPERN, G. Neoliberalismo y migración: los paraguayos en la Argentina en los 90. Política y Cultura: Revista de Universidad Autónoma Mexicana Xochimilco, México, v. 23, p. 67-82, 2005.

HAMEL, R. E. Indigenous languages loss in México: the process of language displacement in verbal interaction. In: FASE,W.; JASPAERT, Kron (Ed.). The state of minority languages. Lisse: Zwts \& Zetlinger, 1995. p. 153-172.

. Conflicto entre lenguas, discursos y culturas en México indígena: ¿la apropiación de lo ajeno y la enajenación de lo propio? In: KLESING-REMPEL, U. (Ed.) Lo propio y lo ajeno; interculturalidad y sociedad multicultural. México: Plaza y Valdés, 1996. p. 149-189.

. Language conflict and language shift: a sociolinguistic framework for linguistic human right. International J ournal of the Sociology of Language: Linguistic Human Rights from a Sociolinguistic Perspective, v. 127, p. 105-134, 1997.

HANKS, W. Language and communicative practices. Boulder: Westview Press, 1996.

HILL, J . Structure and practice in language shift. In: HYLTENSTAM, K.; VIBERG, A. (Ed.) Progression and regression in language: sociocultural, neuropsychological and linguistic perspectives. Cambridge: Cambridge University Press, 1993. p. 58-93.

HILL, J ; HILL, K. Hablando mexicano; la dinámica de una lengua sincrética en el centro de México. México: CIESAS, 1999.

HYMES, D. Models of the interaction of language and social life. In: GUMPERZ, J .; HYMES, D. (Ed.). Directions in sociolinguistics: the ethnography of communication. New York: Holt, Rinehart and Winston, 1972. p. 31-71.

KOOPMANS, R. Deutschland und seine einwanderer: ein gespaltenes Verhältnis. In: KAASE, M.; SCHMIDT, G. (Ed.). Eine lernende demokratie. Gütersloh: BertelmannStiftung, 1999. p. 165-198,

KOOPMANS, R. Partizipation der migranten, staatsbürgerschaft und demokratie: nationale und lokale perspektiven. In: PRÖHL, M.; HARTMANN, H. (Ed.). Strategien der integration. Berlin, 2000. p. 103-111. 
KULICK, D. Language shift and cultural reproduction; Socialization, self, and syncretism in a Papua New Guinean village. Cambridge: Cambridge University Press, 1992.

MALINOWSKI, B. Introducción. In: ORTIZ, F. Contrapunto cubano del tabaco y el azúcar. Caracas: Ayacucho, 1985.

MIGNOLO, W. Palabras pronunciadas con el corazón caliente: teorías del habla, del discurso y de la escritura. In: PIZARRO, A. Palavra, literatura e cultura: a situação colonial. Campinas: UNICAMP, 1993. v. 1, p. 527-562,

PRATT, M. L. Linguistic utopias. In: FABB, N.; ATTRIDGE, D.; DURANT, A.; McCABE, C. (Ed.). The linguistics of writing. Manchester: Manchester UP, 1987. p. 48-66.

PRATT, M. L. Ojos imperiales: literatura de viajes y transculturación. Buenos Aires: Universidad Nacional de Quilmas, 1997.

REGALSKY, P. Territorio e interculturalidad: la participación campesina indígena y la reconfiguración del espacio andino rural. In: LÓPEZ, L. E.; REGALSKY, P. (Ed.). Movimientos indígenas y Estado en Bolivia. La Paz: PROEIBAndes-CENDA-Plural, 2005. p. 107-141.

SAID, E. Orientalism. NewYork: Random House, 1978.

SEGATO, R. La Nación y sus otros: raza, etnicidad y diversidad religiosa en tiempos de diversidad. Buenos Aires: Prometeo, 2007. p. 37-69.

SILVERSTEIN, M. Metapragmatic discourse and metapragmatic function. In: LUCY, J . (Ed.). Reflexive language: reported speech and metapragmatics. Cambridge: Cambridge University Press, 1993. p. 33-60.

SPIVAK, G. The post-colonial critic: interviews, strategies, dialogues. New York, London: Routledge, 1990.

TAYLOR, C. Multiculturalism. Examining the politics of recognition. Princeton (NJ ): Princeton University Press, 1994.

THOMASON, S. G.; KAUFMAN, T. Language contact, creolization and genetic linguistics. Berkeley, Los Angeles: University of California Press, 1988.

TODOROV, T. La conquista de América: el problema del Otro. México: Siglo XXI, 1992.

TURNER, V. La selva de los símbolos; aspectos del ritual ndembu. Madrid: Siglo XXI Editores, 1980.

VIOR, E. Los bolivianos en Buenos Aires fortalecen la democracia. Derechos humanos, inmigración y participación democrática. In: WEHR, I. (Ed.). Un continente en movimiento: migraciones en América Latina. Madrid, Francfort: Iberoamericana,Vervuert, 2006. p. 433-450.

- ¿Cambia la visión de los derechos humanos de una cultura a otra? In: LERTTORA MENDOZA, C. A. (Coord.). Evolución de las ideas filosóficas 1980-2005: XIII J ornadas de pensamiento filosófico. Buenos Aires: FEPAI, 2007a. 
. Migración, derechos humanos y orden político desde una perspectiva intercultural. In: ALEMIÁN, C. (Coord.). Las ideas del Siglo XXI: XII J ornadas de pensamiento filosófico, Buenos Aires: FEPAI, 2007b. p. 209-223.

Participación ciudadana de las comunidades de origen inmigrante en la provincia de Río Negro. Revista Nuevo Espacio Público, n. 2/3, p. 165-198, dic. 2009/ 2010a.

El derecho humano a la migración y la ciudadanía intercultural en zonas de frontera intercultural. In: FORNET-BETANCOURT, R. (Hrsg.). Alltagsleben: Ort des Austauschs oder der neuen Kolonialisierung zwischen Nord und Süd - Everyday Life: Space for Interchange or a Space for New Colonization between North and South - Vida cotidiana: Lugar de intercambio o de nueva colonización entre el norte y el sur: Dokumentation des XIV. Internationalen Seminars des Dialogprogramms Nord-Süd, Denktraditionen im Dialog: Studien zur Befreiung und Interkulturalität, Band 31, Aachen: Wissenschaftsverlag Mainz, 2010b. p. 357-357.

VIOR, E.; BONILLA, A. El derecho humano a la migración y las ciudadanías interculturales emergentes: el caso de la minoría de origen boliviano en la Ciudad de Buenos Aires. CONGRESO DE ANTROPOLOGÍA SOCIAL: FRONTERAS DE LA ANTROPOLOGÍA: 10., 2008, Posadas. Mesa de trabajo: Antropología delas migraciones contemporáneas en la Argentina. Posadas, 2008. CD-Rom.

VIOR, E. et al. Proyecto de investigación UNRN 25/ 09: Condiciones y consecuencias políticas e institucionales de la participación ciudadana de las comunidades de origen inmigrante en la Provincia de Río Negro desde una aproximación intercultural a los derechos humanos. Viedma-Bariloche-Gral. Roca: Universidad Nacional de Río Negro, 2009.

WILLIAMS, R. Marxismo y literatura. Barcelona: Península, 1997.

YUVAL-DAVIS, N. Gender and nation. London: Sage, 1997.

Colaboração recebida em 05/ 04/ 2011 e aprovada em 04/ 06/ 2011. 